\title{
Live-cell Imaging Using Fluorescence Microscopy
}

\author{
Vera DesMarais ${ }^{1}$ \\ 1. Department of Anatomy and Structural Biology, Albert Einstein College of Medicine, Bronx, NY, \\ USA
}

The ever increasing abundance of different fluorescent proteins and vital dyes that are available to researchers today make it possible to study a large variety of dynamic biological processes in live-cells $[1,2]$. Many proteins of interest can be studied by expressing fluorescent fusion proteins in cells, either transiently by transfection or viral infection or permanently using transgenic animals. In addition, many organelles and cellular structures can be fluorescently labeled using specific vital dyes that are able to freely permeate the membranes of living cells. Using these labeling strategies in live cells enables researchers to study the dynamics of proteins in their native environment. In addition to gaining insight into protein dynamics, live imaging also decreases the introduction of fixation artifacts.

Many modern, commercially available microscopes are equipped with hardware to streamline live-cell acquisition. For fast acquisition, it is advantageous to use filter wheels for excitation and emission filters instead of a traditional filter cube turrets, because this allows for rapid alternation of several fluorescent channels throughout image acquisition. Programmable stages have features such as multipoint visiting, allowing for the acquisition of several fields of view over time, thus allowing acquisition of many movies in parallel. In addition, many microscopes are equipped with autofocus modules or z-stacking capacity to compensate for focal drift that may occur during long time-lapse acquisitions. All of these components are usually integrated into the acquisition software on the microscope to allow for a streamlined set up of the experiment.

Live-cell imaging can be achieved either with epi-fluorescent microscopy or confocal microscopy. Epi-fluorescent microscopes that are used for live-cell imaging may use high-sensitivity cameras such as EMCCD cameras for imaging cells using low levels of light and low fluorescent protein expression levels. Epi-fluorescent microscopy can also be combined with deconvolution to generate high-quality images. Another option for live-cell imaging is confocal fluorescent microscopy. While point-scanning confocal microscopes generate the highest quality images, their acquisition speed tends to be rather slow, not allowing the capture of fast, dynamic processes. The better choice for live-cell imaging, especially for rapid intracellular events, would be either a spinning disk confocal or a line-scanning confocal microscope.

Live-cell imaging can be used to study a large variety of different cellular processes. Some cellular processes, such as endocytosis or microtubule and actin dynamics (Figure 1) occur on the scale of seconds or minutes. Other processes such as cell division, wound healing or cell motility might occur over hours or days. It is important to consider the time-scale of the events in question when setting up a live-cell experiment, in order to choose the correct duration of the experiment as well as the time-interval needed to capture the dynamic process.

Beyond the acquisition of basic time-lapse movies, there are advanced imaging techniques that take advantage of the fact that live imaging is a dynamic process. These techniques include fluorescence recovery after photobleaching (FRAP) and chemotaxis experiments. 
In addition to the process of imaging live cells, it is usually also important to be able to analyze the dynamics of the process in question. There are many commercial software packages available that allow for analysis of typical cell motility parameters such as cell speed, direction of movement, etc. as well as particle tracking.
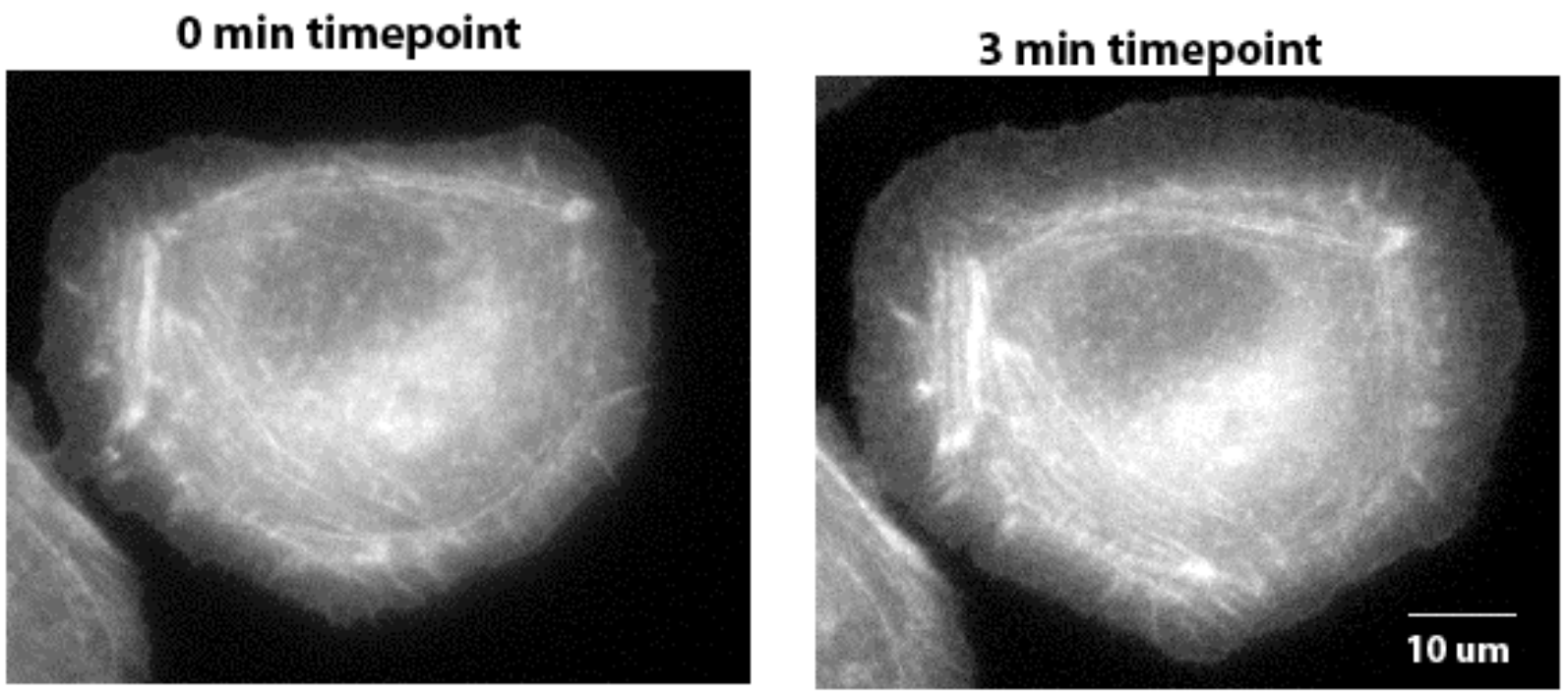

Figure 1. Lamellipod extension and GHP-actın dynamics in adenocarcinoma cells with EGF stımulation [3]. MTLn3 adenocarcinoma cells were transfected with GFP-actin. Time-lapse movies were taken using an inverted Olympus IX70 epi-fluorescent microscope with a 60x oil objective, NA=1.4. During the time-lapse, EGF was added to induce actin polymerization at the leading edge and subsequent lamellipod extension. Images were captured every $10 \mathrm{sec}$, for a total duration of $3 \mathrm{~min}$. This figure shows the same cell at the beginning and at the end of the time-lapse experiment.

\section{References:}

[1] OV Stepanenko et al, BioTechniques 51 (2011), p313.

[2] F Progatzky , MJ Dallman and C Lo Celso, Interface Focus 3 (2013), p. 20130001.

[3] M Lorenz, V DesMarais, F Macaluso, R Singer and J Condeelis. Cell Motility and the Cytoskeleton 57 (2004), p 207. 